\title{
Projeto de Antenas Monopolo Planar Para Sistemas UWB
}

\author{
João N. D. Neto, Leonardo W. Pina, Wederson A. dos Santos, José Felipe de Almeida, Ronaldo O. dos Santos. \\ Instituto de Estudos Superiores da Amazônia - IESAM, Av. Governador José Malcher, 1148 - Nazaré \\ José Maria de Melo Pinto \\ Empresa Brasileira de Telecomunicações - Embratel, Av. Beira Mar, S/N
}

\begin{abstract}
Resumo - O presente trabalho tem por objetivo analisar antenas monopolo planar banda ultra larga. Para isso desenvolveu-se um programa computacional, em Fortran, baseado no método das diferenças finitas no domínio do tempo, método FDTD. Inicialmente, verificou-se a validade do código desenvolvido, através de comparações com resultados simulados disponíveis na literatura. Posteriormente construiu-se duas antenas monopolos e compararam-se os resultados. Depois, analisou-se a relação W/H (largura da antena pela altura) em função da frequiência. $O$ objetivo desta análise era observar que relação apresenta maior largura de banda. Os resultados encontrados são bastante interessantes e servirão para o projeto desse tipo de antenas.
\end{abstract}

Palavras-chaves - Antenas banda ultra larga, método FDTD, perdas de retorno, Antena monopolo planar.

Abstract - The present work has for objective to analyze a planar monopole antennas ultra wideband. In order, based on FDTD method, a Fortran code was developed. First, it was verified validity of the developed code, through comparisons to results simulated and in literature available. Later, two monopole antennas are constructed and had compared the results. There is observed that in high frequencies it has divergence in the results. Finally, the relation W/H (antenna width to its height) to frequency was analyzed. The objective of this analysis is to observe what relation present bandwidth so greater. The joined results are sufficiently interesting and will serve for these antennas project.

Key Words - Ultra Wide Band Antenna, FDTD Method, Return Loss, Planar Monopole Antenna.

\section{INTRODUÇÃO}

Sistemas de comunicações wireless banda ultra larga, sistemas $\boldsymbol{U l t r a}$ Wide $\boldsymbol{B}$ and, usualmente transmitem e recebem pulsos modulados, ocupando largura de banda que vai de aproximadamente de 3.1 a $10.6 \mathrm{GHz}$ [1]. Antenas usadas para aplicações UWB necessitam que parâmetros como ganho e impedância operem em uma larga faixa de freqüência. As antenas monopolo planar de várias configurações [2-5], Antenas de Vivaldi [6] e antenas cornetas TEM [7] são algumas das antenas largamente utilizadas em aplicações UWB.

A utilização de antenas monopolo planar em sistemas UWB é promissor, pois este tipo de antena possui uma estrutura bastante simples e de fácil fabricação, consequentemente, se

J. N. D. Neto, jndn@telecom.iesam-pa.edu.br, L. W. Pina, lwp@telecom.iesam-pa.edu.br, W. A. Santos, wads@telecom.iesampa.edu.br, R. O. Santos, ronaldo@prof.iesam-pa.edu.br, J. M. M. Pinto, jmmelo@embratel.com.br. traduzindo em antenas mais baratas. Entretanto, dos trabalhos analisados [2-5] percebe-se que os mesmos não trazem informação de que relação W/H (ver Figura 1) permite maximizar a largura de banda da antena. Isso porque, pode-se ter uma antena que apresente uma pequena largura de banda ou uma grande largura de banda dependendo da relação W/H. Em [2] observa-se que à medida que a relação varia, ou seja, aumenta, tem-se um aumento na largura de banda, até um ponto em que, mesmo aumentando-se a relação, a largura de banda começa a diminuir. Não fica claro qual a melhor relação W/H máxima para a largura de banda da antena monopolo planar.

Com o objetivo de se analisar a influência da relação W/H na largura de banda da antena monopolo planar, numa faixa de freqüência que abranja a utilizada em sistemas UWB, simulou-se as perdas de retorno para vários valores $\mathrm{W} / \mathrm{H}$. A partir dos resultados, obteve-se a largura de banda em função da relação $\mathrm{W} / \mathrm{H}$.

Para resolver o problema acima, as perdas de retorno, e consequentemente, a largura de banda da antena monopolo planar foram calculadas utilizando o método FDTD [8]. O método FDTD foi utilizado em conjunto com a UPML (Uniaxial Pefectly Matched Layer) [8], para que fosse possível simular radiação na área de análise sem a presença de ondas refletidas.

\section{ANÁliSE DE ANTENAS MONOPOLO PLANAR}

Inicialmente foram analisadas duas antenas monopolo planar, de acordo com a referência [2]. A análise dessas antenas tinha como objetivo verificar a precisão do código desenvolvido, além do, comparar os resultados simulados com os medidos.

Os modelos de análise das antenas são mostrados nas Figuras 1 e 3 . A altura da antena monopolo é $H=20 \mathrm{~mm}$, a largura $W=12 \mathrm{~mm}$ e o comprimento do ponto de alimentação é $g=1 \mathrm{~mm}$.

As características das antenas são simuladas usando o método FDTD-3D. Os parâmetros do método para a análise das antenas são mostrados na Tabela I.

Tabela I - Parâmetros para simulação da antena utilizando o Método FDTD.
\begin{tabular}{|l|l|}
\hline Tamanho das Células & $\Delta \mathrm{x}=\Delta \mathrm{y}=1 \mathrm{~mm} ; \Delta \mathrm{z}=0.5 \mathrm{~mm}$. \\
\hline Iterações & 5000 \\
\hline Onda Incidente & Pulso Gaussiano \\
\hline A. B. C. & UPML 8 Camadas \\
\hline
\end{tabular}

A Figura 2 mostra a perda de retorno da antena monopolo planar mostrada na Figura. 1. A altura do monopolo é de 
$\mathrm{H}=20 \mathrm{~mm}$ para uma freqüência de ressonância de $3.5 \mathrm{GHz}$. Como se pode observar, este tipo de antena possui largura de banda maior que a antena monopolo de fio. Para os dados simulados, obteve-se uma largura de banda, para um nível de perdas de retorno igual a $-10 \mathrm{~dB}$, que vai de $3.0 \mathrm{GHz}$ até 6.4 $\mathrm{GHz}$, ou seja, $3.4 \mathrm{GHz}$. Observa-se ainda, da Figura 2, excelente concordância dos resultados simulados com os da referência (também simulados) [2]. Com relação ao resultado medido houve uma pequena discordância em relação ao resultado simulado. Acredita-se que esta discordância esteja relacionada com as características do material utilizado para a confecção da antena monopolo planar.

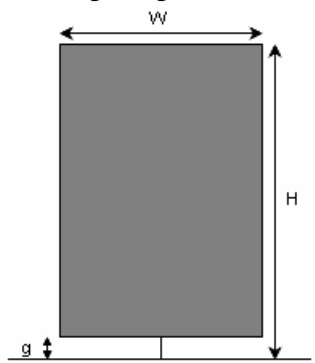

Figura 1. Antena Monopolo Planar Retangular.

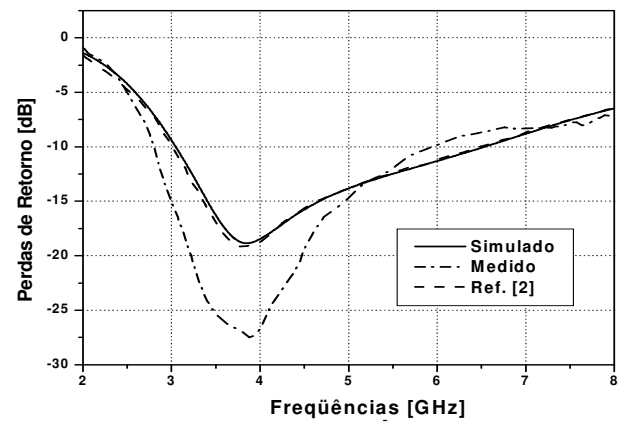

Figura 2. Características de perda de retorno para $\mathrm{W} / \mathrm{H}=0.6 \mathrm{~W}=12 \mathrm{~mm}$ e $\mathrm{H}=20 \mathrm{~mm}, \mathrm{~g}=1 \mathrm{~mm}$

$\mathrm{Na}$ Figura 3, mostra-se uma antena monopolo planar modificada, de acordo com [2]. Observa-se que esta antena consiste de duas antenas monopolo retangular, conectadas por um pino, de comprimento z. A altura da antena monopolo planar é $\mathrm{H}=20 \mathrm{~mm}$, a largura $\mathrm{W}=12, \mathrm{Hu}=2 \mathrm{~mm}, \mathrm{z}=4 \mathrm{~mm}$ e $\mathrm{g}=1 \mathrm{~mm}$. Esta configuração permite a eliminação de radiação nas faixas de freqüências de 5-6 GHz, como podem ser observados na Figura 4, criando dessa forma uma antena com características de freqüência dual, ou seja, pode transmitir e receber em duas freqüências. Esta característica é desejada, para que se evitem interferências ocasionadas pelas redes WLAN, que operam em aproximadamente 5-6 GHz [2]. Em cada freqüência de ressonância, esta antena possui características banda larga.

Com relação à precisão dos resultados, para a antena monopolo planar modificada, observa-se na Figura 4 que os resultados simulados concordam perfeitamente com os disponíveis na literatura [2]. Entretanto, há grande deslocamento nas altas frequiências, considerando os resultados medidos. Novamente acredita-se que este resultado é decorrente das características do material.

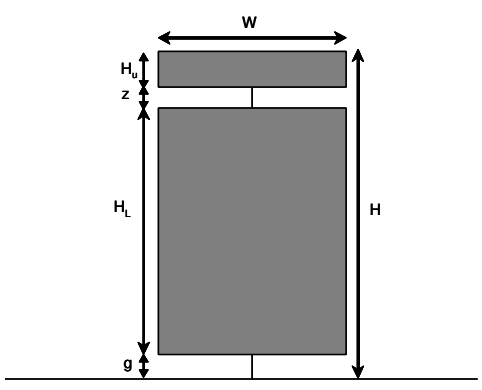

Figura 3. Antena Monopolo Planar Retangular com dupla freqüência de ressonância.

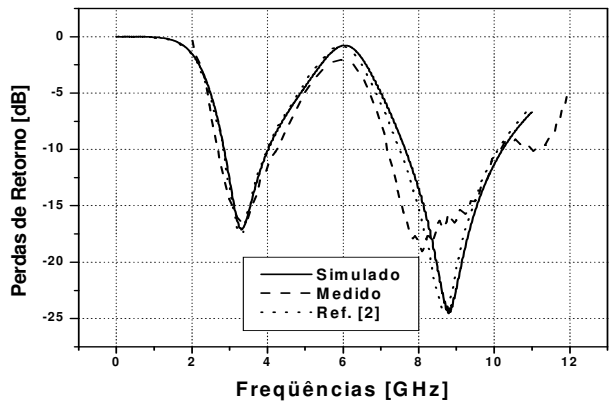

Figura 4. Características de perda de retorno para $\mathrm{W} / \mathrm{H}=0.6 . \mathrm{W}=12 \mathrm{~mm}$ $\mathrm{H}=20 \mathrm{~mm}, \mathrm{H}_{\mathrm{L}}=13 \mathrm{~mm}, \mathrm{z}=4 \mathrm{~mm}$ e $\mathrm{g}=1 \mathrm{~mm}$.

\section{CURVAS PARA PROJETO DE ANTENA MONOPOLO PLANAR UWB}

No projeto de antenas monopolo planar UWB (Fig. 1), um fator que precisa ser esclarecido é, qual a melhor relação W/H para a maximização da sua largura de banda? Para se responder a esta pergunta, simularam-se várias antenas, ou seja, variou-se a relação $\mathrm{W} / \mathrm{H}$ de $0.1-1$, assim como a faixa de freqüência, de $1-10 \mathrm{GHz}$, cobrindo-se, desta forma, a faixa de freqüência de sistemas UWB. Os valores de perda de retorno obtidos foram, então, tratados utilizando a seguinte equação [9]:

$$
B W(\%)=2 \frac{f_{H}-f_{L}}{f_{H}+f_{L}},
$$

onde $f_{H}$ representa a maior frequiência, $f_{L}$ representa a menor frequiência. A largura de banda foi definida para uma perda de retorno igual a $-10 \mathrm{~dB}$. Além disso considerou-se um valor de $-14 \mathrm{~dB}$ como um bom casamento de impedância [10]. Isso significa que foram utilizadas apenas as curvas de perda de retorno que apresentam pelo ao menos uma perda de retorno de $-14 \mathrm{~dB}$, na freqüência de análise.

Nas Figuras 5 e 6, mostram-se o comportamento da largura de banda em função da frequiência e da relação $\mathrm{W} / \mathrm{H}$, respectivamente. Na Figura 5 observa-se que para uma relação $\mathrm{W} / \mathrm{H}$ de $0.5,0.6$ e 0.7, obtêm-se as maiores largura de banda, para uma faixa de freqüência de $2-9 \mathrm{GHz}$. Na Figura 6 observa-se que para uma relação de $\mathrm{W} / \mathrm{H}$ de $0.1-0.4$, não se consegue uma largura de banda maior que $40 \%$. Para uma relação W/H de 0.6 e 0.7 , obtêm-se as maiores larguras de banda, para as seguintes faixas de freqüências, de $7-9 \mathrm{GHz}$. Todos os resultados foram obtidos de curvas de perda de retorno, como se pode observar da Figura 7. Observa-se desta 
figura 7 que a medida que se aumenta a relação $\mathrm{W} / \mathrm{H}$, aumenta-se a largura de banda da antena analisada.

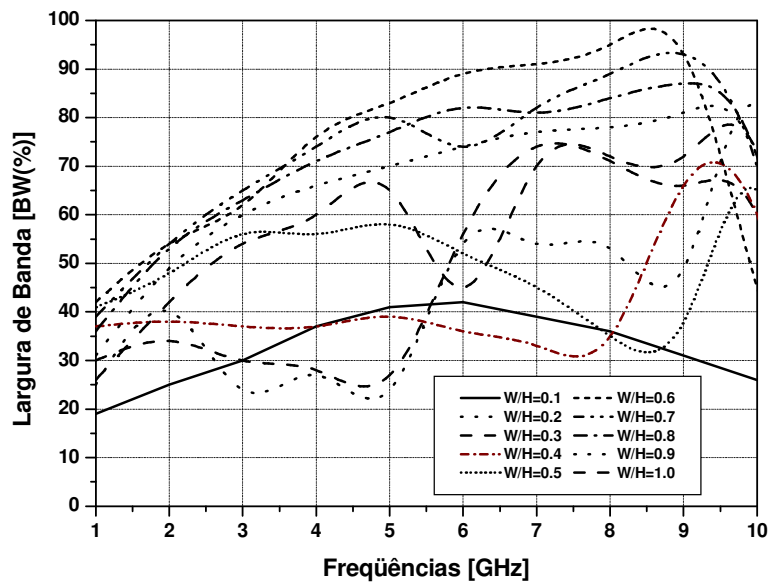

Figura 5. Curvas de largura de banda em função da frequiência, para vários valores da relação W/H, para a Antena Monopolo Planar Retangular.

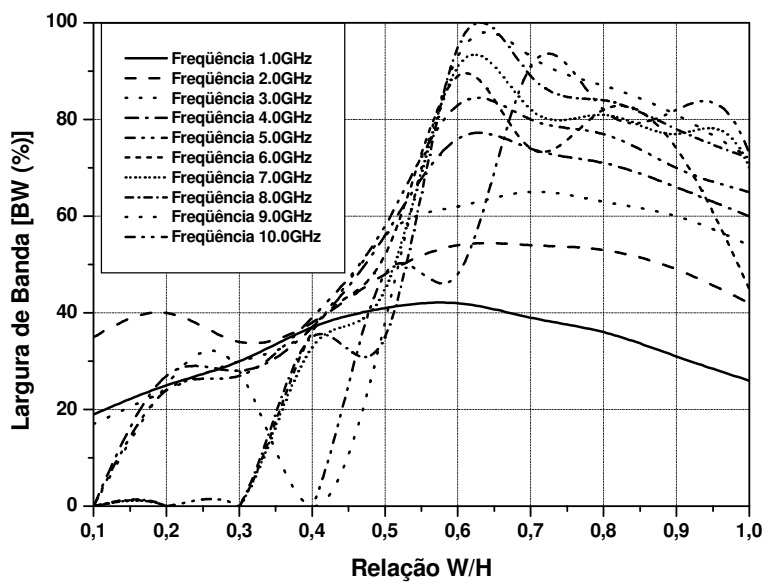

Figura 6. Curvas de largura de banda em função da relação W/H, para vários valores de freqüência, para a Antena Monopolo Planar Retangular.

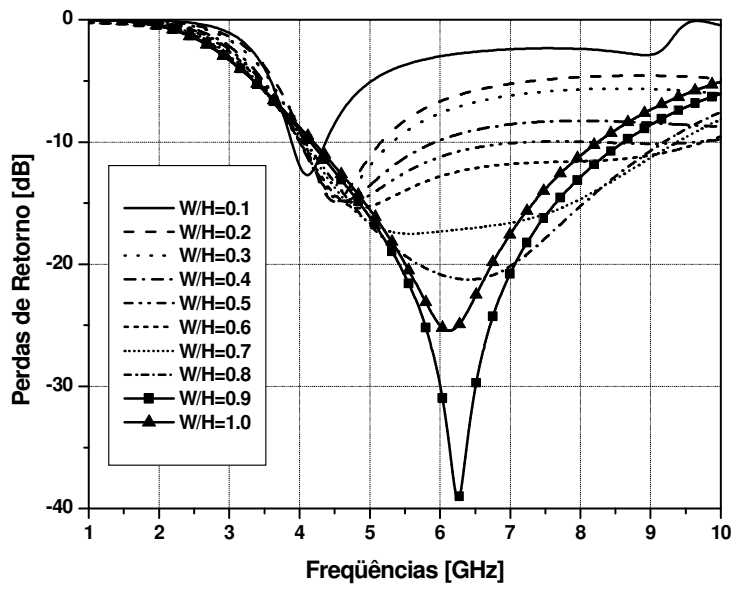

Figura 7. Curvas de perdas de retorno, para uma Antena Monopolo Planar, projetada para ressoar em $5 \mathrm{GHz}$.

Na Figura 7, os resultados apresentados são de uma antena monopolo projetada para possuir uma frequiência de ressonância em $5 \mathrm{GHz}$. Um resultado bastante interessante é que há um deslocamento da freqüência de ressonância à medida que a relação $\mathrm{W} / \mathrm{H}$ aumenta. Por exemplo, o menor valor de perda de retorno, aproximadamente $-40 \mathrm{~dB}$, para $\mathrm{W} / \mathrm{H}=1$, é encontrado para a freqüência de $6.22 \mathrm{GHz}$. Entretanto, na frequiência de $5 \mathrm{GHz}$, pode-se observar que a perda de retorno é de aproximadamente $-15 \mathrm{~dB}$, o que é considerado muito bom.

\section{CONCLUSÕES}

Desenvolveu-se um código computacional, em Fortran, para a análise de antenas monopolo planar UWB, usando-se o método FDTD. Para a validação do código, compararam-se os resultados obtidos com os disponíveis na literatura. Observou-se excelente concordância entre os resultados simulados. A partir dos resultados simulados construíram-se duas antenas. Os resultados obtidos da medição dessas antenas foram comparados com os simulados. Observou-se que em altas freqüências houve deslocamento da freqüência de ressonância. Acredita-se que este deslocamento esteja relacionado com o tipo de material utilizado para construir a antena em questão.

Observou-se, através das simulações que a largura de banda da antena monopolo planar varia com a relação $\mathrm{W} / \mathrm{H}$, ou seja, quanto maior a relação, maior a largura de banda. A partir dos resultados, observou-se que a relação $\mathrm{W} / \mathrm{H}$ que maximiza a largura de banda, está compreendido entre 0.6 e 07 , ou seja, $0.6<\mathrm{W} / \mathrm{H}<0.7$, para a faixa de freqüência utilizada em sistema UWB, 3.1-10. GHz.

As antenas monopolo planar retangular devido sua fácil fabricação, baixo custo, são bastante promissoras para serem utilizadas em sistemas Wireless UWB. Dessa forma, escolhendo o material apropriado, consegue-se produzir antenas com excelente qualidade e precisão.

\section{REFERENCIAS}

[1] S. Gupta, M. Ramesh and A. T. Kalghatgi, "Design of Optimizd CPW fed Monopole Antenna for UWB Applications," IEEE APM2005 Proceedings.

[2] Y. Rikuta and R. Kohno, "Planar Monopole Antenna With Dual Frequency for UWB System,"IEEE Trans. Antennas Propagation, pp. $176-179,2003$.

[3] Z. N. Chen, X. H. Wu, N. Yang and M. Y. W. Chia, "Design Consideration for Antennas in UWB Wireless Comumnication System, "in IEEE AP-S Int. Symp. Dig., pp. 822-825 June 2003

[4] A. Kerkhoff and H. Ling, "Design of a Planar Monopolo Antenna for Use with Ultra-Wideband (UWB) Having a Band- Notched Characteristic", IEEE Symposium on Antennas and Propagation, Columbs, $\mathrm{OH}$, vol. 1 pp. 830-833, June, 2003

[5] Y. Lim, H. Kuk, Y. J. Yonn and Young-Hwan Kim, "The Design of Planar and Rolled Fat Monopole Antenna for UWB Radio System,"IEEE APM2005 Proceedings.

[6] E. Guillanton, J. Y. Dauvignac, C. Pichot, and J. Cashman, "A New Design Tapered Slot Antenna for Ultra-Wide Band Applications", Microwave and Optical Technology Letters 19, pp.286-289, 1998.

[7] X. Li, S. C. Hagness, M. K. Choi, and D. W. V. Weid, "Numerical and Experimental Investigation of an Ultrawide Ridged Pyramidal Horn Antenna With Curved Lauchhing Plane for Pulse Radiation", IEEE Antenna and Wireless Propagation Letters, vol. 2, pp. 259-262, 2003.

[8] A. Taflove and S. C. Hagness: Computational eletrodynamics: The finitedifference time-domain Method, Artech house,inc., Boston, 2000.

[9] H. Schantz, The Art and Science of ULTRAWIDEBAND ANTENNAS, Artech House, 1955

[10] R. E. Collin, Fondations For Microwave Engineering, 2, Mcgraw-Hill International Edition 1992. 